\title{
Targeting Nuclear Receptors with Lentivirus-Delivered Small RNAs in Primary Human Hepatocytes
}

\author{
Maria Thomas Jessica K. Rieger Benjamin A. Kandel Kathrin Klein \\ Ulrich M. Zanger \\ Dr. Margarete Fischer-Bosch Institute of Clinical Pharmacology, Stuttgart, Germany
}

\section{Key Words}

Primary human hepatocytes $\bullet$ Nuclear receptors $\bullet$ RNAi $\bullet$ Lentivirus $\bullet$ CAR $\bullet$ PPAR $\alpha \cdot$ miR-143

\begin{abstract}
Background: RNA interference (RNAi) has tremendous potential for investigating gene function and for developing new therapies. Primary human hepatocytes $(\mathrm{PHH})$ are the "gold standard" for studying the regulation of hepatic metabolism in vitro. However, application of RNAi in PHH has some technical hurdles. The objective of this study was to develop effective and robust protocol for transduction of PHH with lentiviral vectors. Methods: We used lentiviral vectors to transduce PHH for introduction of short hairpin RNAs (shRNAs) targeting constitutive androstane receptor (CAR), peroxisome proliferator activated receptor alpha (PPAR $\alpha$ ), and microRNA, miR-143. Infection efficiency was quantitatively analyzed by flow cytometry and microscopy. Target gene expression was assessed using quantitative realtime (qRT-PCR) method. Results: Lentiviral vector transduction resulted in $\geq 95 \%$ of infected cells at low multiplicity of infection (MOI) of 3, which did not impair cellular viability. We demonstrated the feasibility of this technique in studies on targeting nuclear receptors, PPAR $\alpha$ and CAR, with shRNAs as well as in lentivirus-mediated overexpression and knock-down of miRNA-143 experiments. Conclusions: We developed an efficient and robust protocol with standardized procedures for virus production, method of titer determination, and infection procedure for RNAi in primary human hepatocytes based on delivery of shRNAs, microRNAs or anti-microRNAs in different laboratory settings. This approach should be useful to study not only the regulation via nuclear receptors but also other biological, pharmacological, and toxicological aspects of drug metabolism.
\end{abstract}

M. Thomas and J.K. Rieger contributed equally to this work. 


\section{Introduction}

RNA interference (RNAi) is a powerful approach for reducing expression of endogenously expressed proteins [1]. It is widely used in biological research as well as for therapeutic applications [2]. Various methods, including delivery of small interfering RNAs (siRNAs) and expression of small hairpin (shRNAs) RNAs from viral vectors, have been developed for RNAi in cell culture and in vivo. Nevertheless, various complex barriers for achieving efficient RNAi have become evident. These hurdles include: specificity for the target gene, delivery to the correct cell or tissues, the durability of RNAi activity, and considerations of the stability of the target mRNA and encoded protein. Particular challenges concern the application of RNAi in primary cells, where conventional transfection techniques by lipophilic agents result in low transfection efficiency.

Primary human hepatocytes (PHH) are the "gold standard" of hepatic cell culture models not only for evaluating biotransformation and toxicity of drugs and other xenobiotics in vitro, but also for studying the regulation of hepatic phenotypes by nuclear receptors (NR) and other transcription factors. However, application of RNAi in primary hepatocytes still remains challenging. Although different protocols are available, the efficiency of shRNA delivery is often not satisfactory [3]. Besides low efficiency, a further limitation is low reproducibility between different batches of PHH due to the high variability of the individual donor cells.

One preferred method that allows achieving higher transfection rates is the use of recombinant viruses, in particular adenoviral and lentiviral derivatives. While transductions of PHH with adenoviral vectors are well established, these vectors are non-replicating and remain episomal. [4-6]. Besides that, adenoviral vectors have further limitations which might preclude them from many applications in basic research, including strong immunogenicity and typically short duration of shRNA expression. Additionally, there is evidence that adenoviral vectors may contain properties and/or stimulate cellular processes that block or counteract RNAi-mediated gene silencing [7, 8]. Moreover, since humans commonly come in contact with adenoviruses, which cause respiratory, gastrointestinal and eye infections thereby triggering a rapid immune response, this can in turn cause potential unspecific results in the study of $\mathrm{PHH}$ [9].

By contrast, retroviral vectors integrate into the host genome, but most of them require cell division for integration event to occur [10].HIV- (human immunodeficiency virus) based vectors are currently the most popular lentiviral-based expression systems [11]. They effectively transduce both dividing and non-dividing cells and stably integrate into the genome of the host cell, thereby facilitating long-term transgene expression. Furthermore, in contrast to adenovirus, lentiviral particles elicit less unspecific effects because they do not lead to an inflammatory response in the cell culture [12].To our surprise, very few publications describe applications using lentivirus for delivery of small RNAs into PHH, presumably because methodologically sound protocols have not been available $[13,14]$. Thus, there is a general lack of validated protocols for the production of lentiviral particles as well as experimental details for the reproducible infection of PHH with lentiviral vectors. The primary aim of this study was therefore to develop robust, comprehensive and reliable protocol for the design, generation and purification of lentiviral particles and their use for transduction of PHH. For protocol validation, we generated a panel of vectors coding for active shRNAs targeting major nuclear receptors as well as known liver-specific microRNAs and anti-microRNAs. We show that lentivirus-delivered shRNA successfully inhibits expression of CAR in PHH, which furthermore resulted in the downregulation of its target genes, CYP2B6 and CYP2C8. Additionally, we demonstrate for the first time that shRNA-mediated knockdown as well as overexpression of miR-143 in PHH influences expression of CAR, CY2B6 and CYP2C8. We have previously used these tools and protocols to demonstrate the significant role of PPAR $\alpha$ in the regulation of several DMEs in the liver $[15,16]$. 


\section{Materials and Methods}

Detailed description of all used reagents is freely available and can be found here: http://seek. virtuelle-leber.de/sops/13.

Chemicals

G418 (Calbiochem \#345812), TurboFect (Fermentas, \#R0531), Trypsin (0.05\%) with EDTA (Gibco Life Technologies \#25300-054), PEG-it ${ }^{\text {TM }}$ Virus Concentration Solution (System Biosciences\#LV810A) were used for the generation of viral particles. For the efficient infection polybrene (Sigma\#H9268) was applied together with lentivirus to PHH.

\section{Culturing media}

Medium for culturing HEK293FT and HT1080 cells: D-MEM (high glucose) (Gibco\#41965-039) with

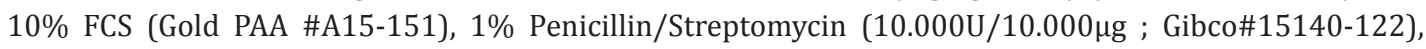
$1 \%$ sodium pyruvate (100mM, Gibco\#11360-039), 1\% glutamine (200 mM-100X, Gibco\#25030-024), 1\% MEM NEAA (non essential amino acids, 100X, without L-glutamine; Gibco\#11140-035). 1\% BSA/PBS: 1\% solution of BSA (Sigma\# A4503-50G) in PBS, sterile filtered, aliquoted and stored at $-20^{\circ} \mathrm{C}$.

\section{Culturing of primary human hepatocytes}

The detailed procedure of hepatocytes isolation and cultivation can be found also elsewhere [17]. Cells were seeded on collagen type I-coated culture dishes at a density of $1 \times 10^{5}$ cells $/ \mathrm{cm} 2$ and cultivated in Williams medium E (Life Technologies, Darmstadt, Germany), supplemented with $10 \%$ fetal calf serum (GIBCO, Karlsbad, USA), 2m m l-glutamine (Life Technologies, Darmstadt, Germany), $100 \mathrm{U} / \mathrm{ml}$ penicillin/100 $\mathrm{mg} / \mathrm{ml}$ streptomycin (GIBCO, Karlsbad, USA), 0.032 I.E./ml insulin (Aventis-Sanofi, Frankfurt, Germany), $0.1 \%$ DMSO, and $0.1 \mathrm{mM}$ dexamethason (Sigma Aldrich, Darmstadt, Germany).

\section{Plasmid constructs}

We have used BLOCK-iT U6 RNAi Entry Vector Kit (Invitrogen \# 49-4400) for cloning shRNAs targeting CAR and PPAR $\alpha$ into pENTR vector. Using Gateway ${ }^{\mathrm{TM}}$ technology of Invitrogen (ViraPower ${ }^{\mathrm{TM}}$ Lentiviral Gateway Expression Kit (Invitrogen\#K49-6000)), the shRNA sequence can be easily shuttled into lentiviral expression vector pLenti6. This vector encompasses also a blasticidin expression cassette for generating stable clones under selection and can be purchased as a component of ViraPower Lentiviral Expression System. For the visual control of infection efficiency a modified vector containing a substitution of blasticidine gene with EGFP expressing cassette was used (kindly provided by M.Kriebel, NMI Reutlingen, Germany). For microRNAs experiments, we have used vectors provided by System Biosciences ${ }^{T M}$ - pMIRNA1 and pmiRZip - which are also compatible with ViraPower ${ }^{\mathrm{TM}}$ lentiviral packaging mix $\left(\mathrm{miRZip}^{\mathrm{TM}}\right.$ Lentivector-based AntiMicroRNAs (System Biosciences \# MZIPxxxPA/AA-1) and pMIRNA1 Lentivector-based microRNAs (System Biosciences \# PMIRHxxxPA/AA-1)).

\section{$L D H$ release measurements}

For the measurement of LDH release, a CytoTox 96® Non-Radioactive Cytotoxicity Assay (G1780) from Promega was used according to the manufacturer's instructions. The percentage of LDH released from hepatocytes was calculated as LDH present in culture medium in relation to total LDH obtained in culture medium and hepatocytes.

Real-time reverse transcription-polymerase chain reaction (RT-PCR)

The procedure has been described previously [16]. For the assessment of transcripts following Taqman Gene Expression Assays from Life Technologies were used - Hs00947536_m1 for PPAR $\alpha$; Hs00901571_m1 for CAR; Hs04183483_g1 for CYP2B6; Hs02383390_s1 forCYP2C8; Hs00604506_m1 forCYP3A4; Mm01264680_ $\mathrm{m} 1$ for miR-143. For the detection of EGFP: forward primer: 5'-AAGCAGCACGACTTCTTCAAGTC-3', reverse primer: 5'- TCGCCCTCGAACTTCACCTC-3', were used in combination with MESA GREEN qPCR MasterMix Plus for SYBRß Assay (Eurogentec, RT-0610-03). 
Western Blot

For the detection of CYP2B6, the antibody from BDGentest (\# 458326) was used in the protocol as described earlier [18].

Flow cytometry FACS analysis

On day three after lentivirus infection, the cells were trypsinized by adding $150 \mu \mathrm{l}$ of trypsin solution per well, let stand for 5-10 minutes and diluted with $850 \mu \mathrm{l}$ of medium. FACS measurement were performed using Becton Dickinson FACS Calibur device counting 10.000 events per probe and evaluated using CELLQuest software.

\section{Sequences}

shRNA sequences CAR: CACCGCAGAAGTGCTTAGATGCTGGCGAACCAGCATCTAAGCACTTCTGC (top); AAAAGCAGAAGTGCTTAGATGCTGGTTCGCCAGCATCTAAGCACTTCTGC (bottom). PPAR $\alpha$ shRNA sequences and non-coding shCTR sequences can be found elsewhere [16].

\section{Results and Discussion}

Generation of silencing constructs and applications of the method

Each experiment requires the design and generation of appropriate gene-specific expression constructs. For instance, BLOCK-iT ${ }^{\mathrm{TM}}$ RNAi Designer tool from Invitrogen (Life Technologies, Darmstadt, Germany) can be used for design of specific and effective shRNA sequences. At least two independent silencing sequences targeting different sites should be used within one mRNA to demonstrate specific knock-down. The designed template sequences encoding short hairpin RNAs, microRNAs or anti-microRNAs are then cloned into the appropriate lentiviral shRNA/microRNA/anti-microRNA expression vector. Alternatively they may be purchased as ready-to use reagents from a variety of vendors.

The described method for viral production and titer determination can be applied to all expression vectors compatible with packaging systems of the second and third generation, i.e. a lentiviral vector with a chimeric 5' LTR in which the HIV promoter is replaced with CMV or RSV, thus making it TAT-independent. A short summary of the protocol is provided in Table 1 and a detailed description can be found at the Virtual Liver Network server (http://seek. virtuelle-leber.de/sops/13.). Examples of these vectors include pLKO.1 (Sigma-Aldrich), pLentiLox3.7 and its modification, pLB, with an added genetic element for the prevention of epigenetic silencing;pSico/pSicoR vectors, which can be used for conditional (Cre-Lox), stable expression of shRNAs for RNA interference in cells and transgenic mice (ATCC); pLenti6 (Invitrogen) and pLove (for Lentiviral Over-Expression) (Addgene).

The protocol for infection was specifically optimised for PHH in monolayer culture. Magnetofection resulted in approx. 20-30\% of positively-transfected cells and was highly dependent on donor [19]. In contrast, lentivirus infected consistently 90-95\% of PHH using a MOI of 3, based on our experience with PHH from more than ten donors (Fig 1A). It is noteworthy to mention that application of MOI higher than 3 did not lead to higher expression of GFP, as observed using qRT-PCR analysis of GFP mRNA expression in PHH (Fig 1B). Accordingly, the infection efficiency as determined using flow cytometry reached $\geq 95 \%$ cells at MOI 3 (Fig. 1C). Two cell lines of hepatic origin, HepG2 and HuH7 and the human epithelial colon cell line LS-174T, could be efficiently infected using the same conditions with an even lower MOI of 2 (data not shown).

Lentiviral infection does not influence viability and functional characteristics of $\mathrm{PHH}$

Our results demonstrated that lentiviral vector transduction itself did not impair primary hepatocytes cellular viability, as measured by cellular release of lactate dehydrogenase (LDH) (Fig. 2). Furthermore, drug oxidation capacity of PHH, as tested by measurement of seven cytochrome P450 enzyme activities by LC-MS/MS [20] remained also unaffected [16]. 


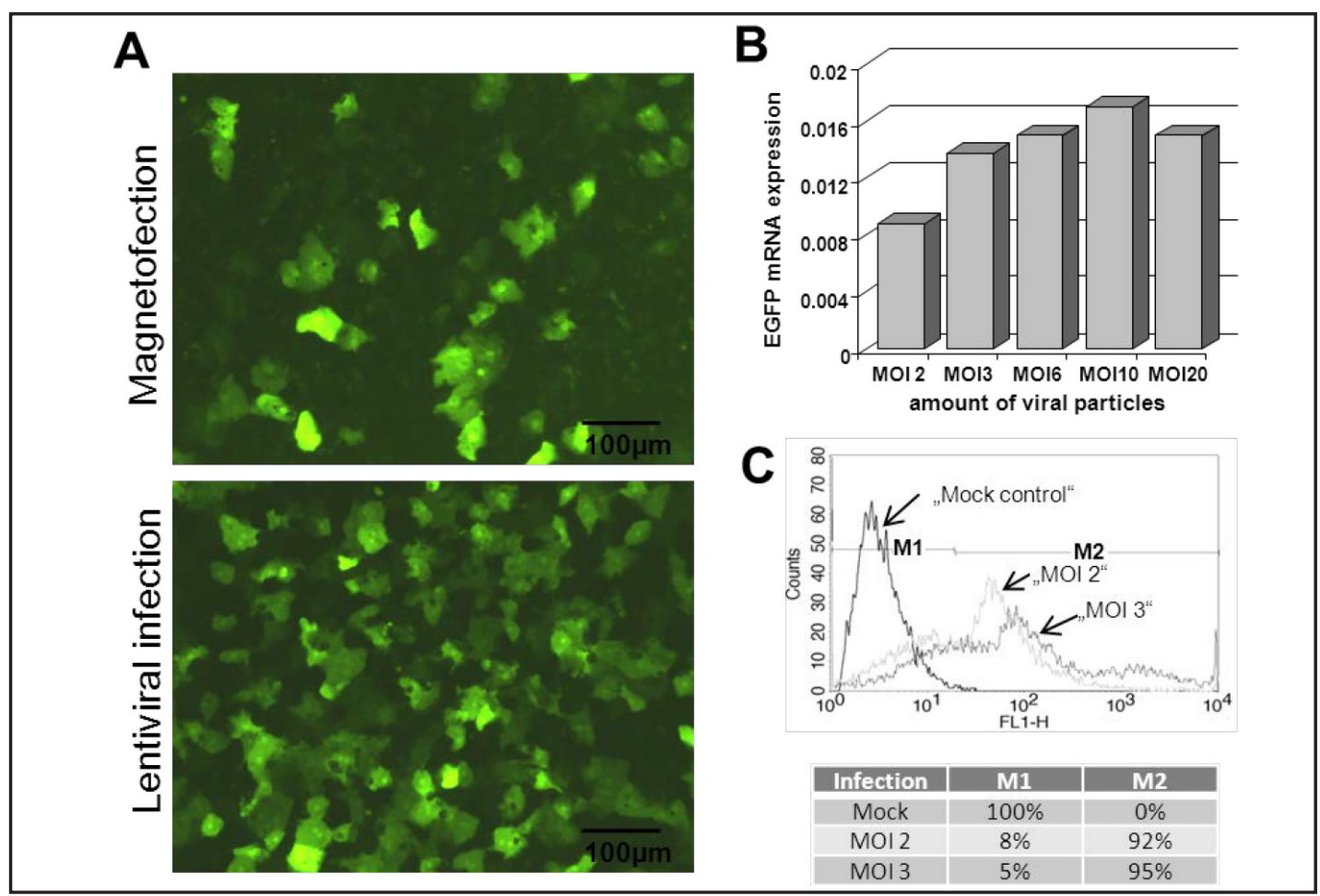

Fig. 1. Lentiviral vectors effectively transduce primary hepatocyte cultures. A: Representative microscopic images (10x) comparing transfection efficiency using magnetofection (upper panel) with lentivirus-mediated infection at MOI=3 (lower panel). B: Quantitative real-time RT-PCR analysis of EGFP expression reflects the accumulation of EGFP transcripts in PHH depending on the amount of viral particles used for infection. The application of MOI=3 is sufficient and enough to infect more than $95 \%$ of PHH for successful knock-down of target gene. C: Analysis of infection efficiency was performed by flow cytometry using a fluorescence activated cell sorter (FACS). The histogram shows the frequency distribution of the markers "M1" and "M2" indicating the intensity of the fluorescence signal from GFP (FL1-H channel). M1 displays the background staining of "mock"-infected (viral particles which do not contain GFP cDNA in the backbone) PHH. The second distribution (M2) illustrates infection efficiency with a GFP-containing lentivirus. The table indicates the quantitative evaluation of flow cytometry measurements using CellQuest software (Becton \& Dickinson).

Lentivirus-mediated knock-down of nuclear receptors CAR and PPAR $\alpha$ using shRNAs leads to the downregulation of their target genes

We constructed specific lentiviral shRNA-vectors to silence the expression of CAR and PPAR $\alpha$ in PHH. As shown in Figure 3A, infection with shRNA-viruses targeting the $5^{\prime}$ (shRNA1) and 3'- (shRNA2) regions in PPAR $\alpha$ led to $>50 \%$ reduction in the expression of PPAR $\alpha$ starting at day four, as compared with cells treated with non-targeting shRNA (shCTR). Similarly, knock-down of CAR consistently resulted in up to $\sim 50 \%$ reduction in mRNA levels of CAR, based on experiments from three independent donors. Furthermore, expression of the CAR downstream target genes, CYP2B6, CYP2C8, CYP3A4, assessed five days following infection, was accordingly suppressed (Fig 3B, light grey bars). In contrast, the activation of CAR using its chemical agonist, CITCO, led to the concomitant upregulation of these genes (Fig. 3B, dark grey bars). The lentivirus-mediated CAR gene silencing, as well as treatment with CITCO, led to the corresponding down - or up- regulation of CYP2B6 on the protein level as compared with shCTR and shown using Western Blot analysis (Fig 3C).

Impact of lentivirus-mediated overexpression and knockdown of miR-143 in PHH

To elaborate potential effects of miRNAs overexpression and knock-down on NRs, we first performed in silico target prediction analysis using our MIRNA-DISTILLER tool [21, 


\section{Cellular Physiology $\quad$ Cell Physiol Biochem 2014;33:2003-2013 and Biochemistry

Fig. 2. Lentiviral infection has no influence on the viability of PHH. Viability of PHH was quantitatively measured as the ability to release lactate dehydrogenase (LDH). For that, PHH were infected with different shRNAs, targeting several nuclear receptors and their target genes, together with non-targeting shRNA control. shRNAs 9a and 9b target NR, which apparently leads to the damage of PHH.

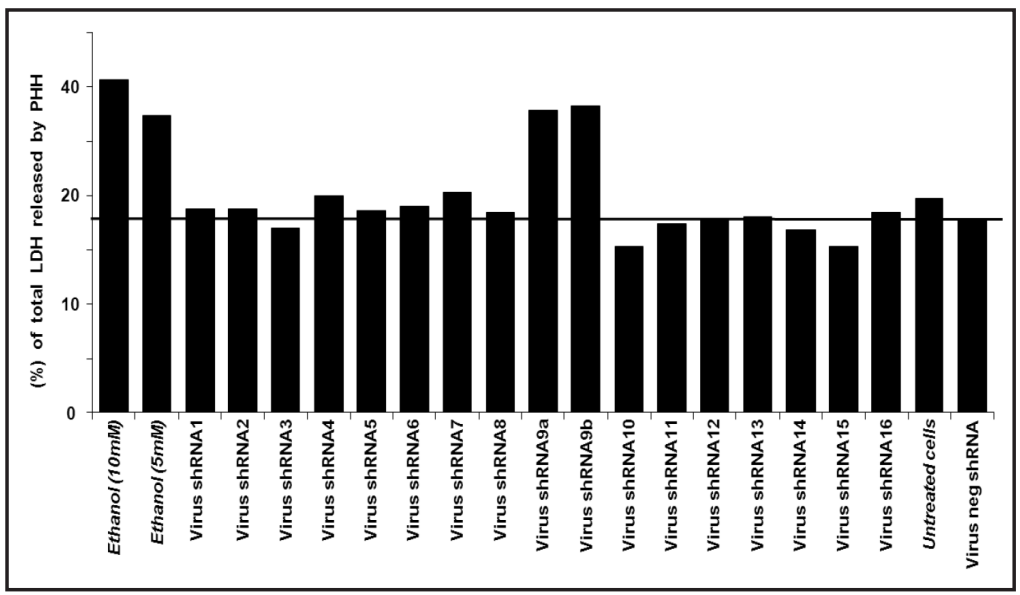

Fig. 3. Lentivirus-mediated knock-down of PPAR $\alpha$ and CAR leads to the downregulation of their target genes. A. Time-resolved qRT-PCR analysis of a human PPAR $\alpha$ in $\mathrm{PHH}$, lysed on the indicated days after infection with lentivirus carrying shRNAs targeting human PPAR $\alpha$ at the 3 ' - (shRNA1) or 5'mRNA end (shRNA2), in comparison to a non-silencing control shRNA (set to 1). The knock-down of this particular target achieves its maximum at day 4 upon infection and stays preserved over cultivating till day 11 . Error bars indicate standard deviation between infection experiments in $\mathrm{PHH}$ cultures of three independent donors. B. Relative mRNA expression of CAR and its target genes in the PHH, either infected with CAR shRNA (light grey bars) or treated with CAR agonist, CITCO (dark grey bars). Error bars for the knock-down experiments indicate standard deviation between infection

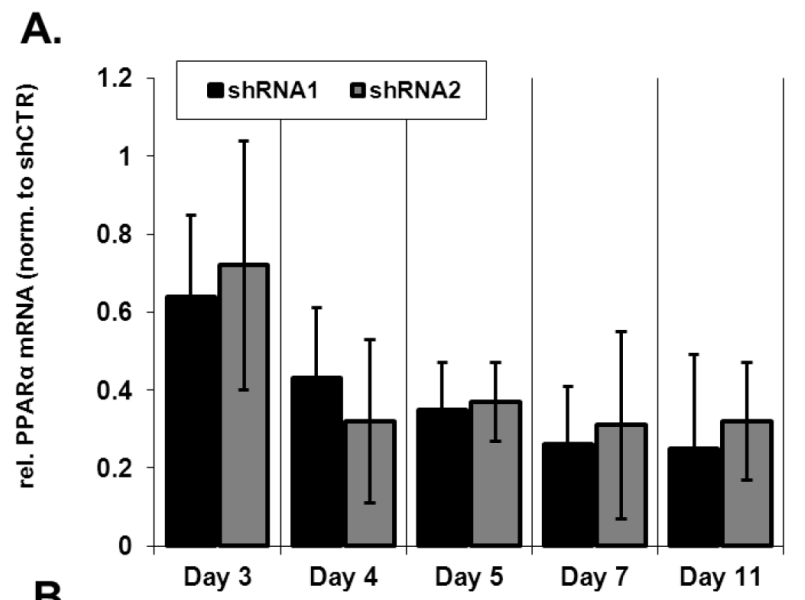

B.

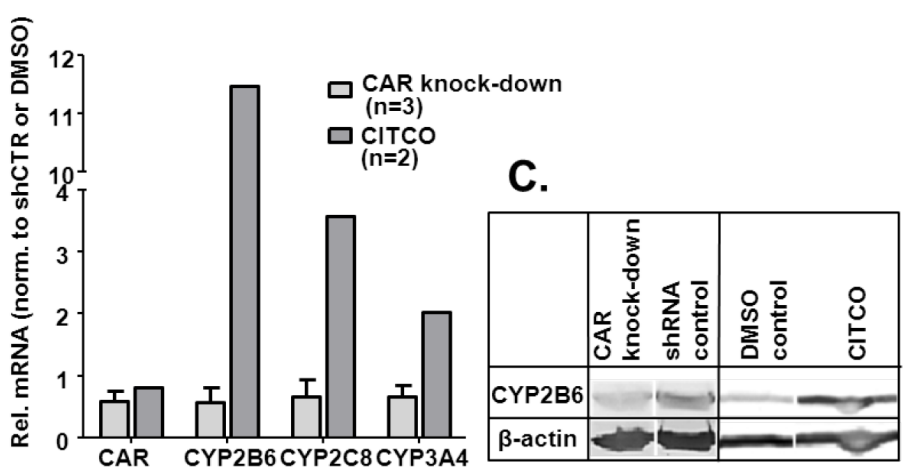

experiments in PHH cultures of three independent donors. For the induction experiments, PHH cultures of only two independent donors were used due to the material limitations. C. Protein expression analysis of CAR target protein, CYP2B6, following knock-down of CAR (left panel) or chemical activation of CAR using $100 \mathrm{nM}$ of CITCO (right panel).

22]. The search for the microRNAs targeting a variety of genes involved in drug metabolism revealed a high score for miR-143, which was therefore chosen for the lentivirus-mediated overexpression and knock-down experiments in PHH. This microRNA was so far discussed in the context of hepatocellular carcinoma (HCC) progression and was found to be fourto eight- fold upregulated in the liver tissues of HCC patients [23]. As shown in Figure 3, overexpression of miR-143 five days after infection resulted in more than 3000-fold 
Thomas et al.: A Lentivirus-Based System to Functionally Silence Nuclear Receptors in Primary Human Hepatocytes

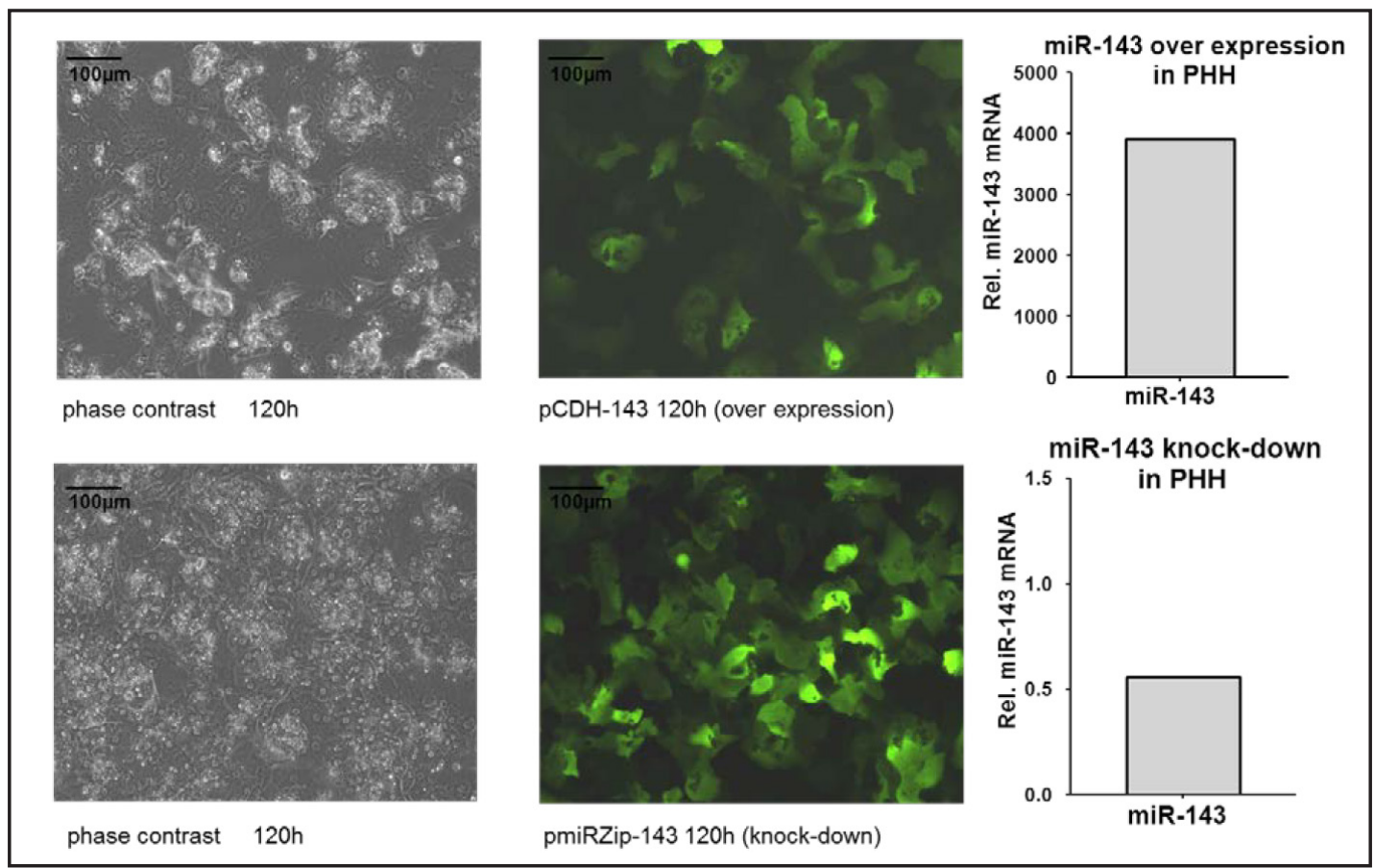

Fig. 4. Efficient lentiviral delivery of miRNA and anti-miRNA constructs into PHH. Representative microscopic images (20x) in phase contrast (left) and fluorescence (right) demonstrating infection efficiency for the miR-143 overexpression (upper panel) and anti-miR-143 expression (bottom panel) five days upon lentivirus treatment at MOI=3. Quantitative real-time RT-PCR analysis of miR-143 expression reflects successful accumulation of miR-143 transcripts in PHH following overexpression (upper right panel) and corresponding knock-down of miR-143 mRNA (bottom right panel) in comparison to the non-targeting control miRNA.

Fig. 5. Efficient lentiviral delivery of miRNA and anti-miRNA constructs into PHH. Relative mRNA expression of CAR and its target genes in the $\mathrm{PHH}$, either following miR-143 overexpression (light grey bars) or after miR-143 knock-down (dark grey bars), both upon 5 days of infection. Error bars for the miR143 overexpression experiments indicate standard deviation between infection experiments in $\mathrm{PHH}$ cultures of three independent donors. For the miR143 knock-down studies, PHH cultures of only two independent donors were used due to the material limitations.

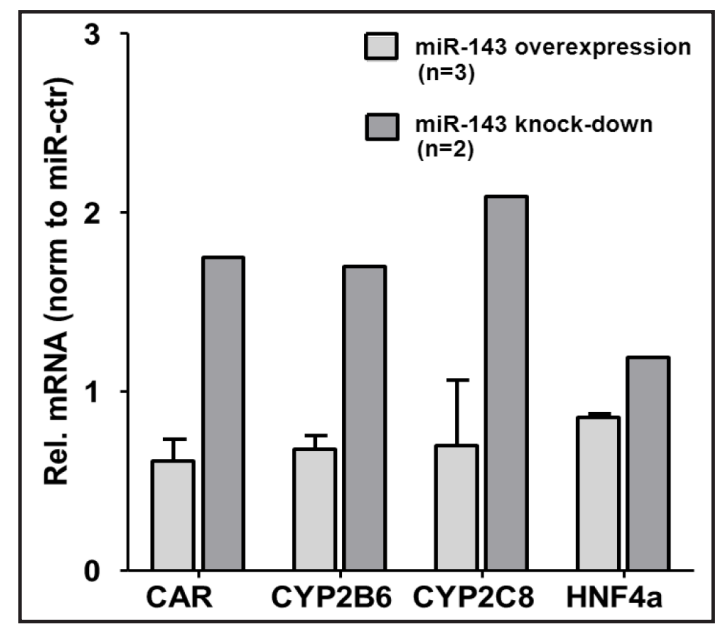

induction of its mRNA (Fig. 4, upper panel). In contrast, lentivirus-mediated downregulation of miR-143 led to 50\% decreased mRNA compared to the non-targeting control (bottom panel). An infection efficiency of over $90 \%$ was determined using flow cytometry I as shown on the representative microscopic images with lentivirus-mediated infections at MOI=3 (Fig. 4). The lentivirus-mediated overexpression of miR-143 in PHH resulted in the downregulation of CAR mRNA with paralleled inhibition of the expression of its target genes, CYP2B6 and CYP2C8, and slight, but consistent, reduction of HNF4a mRNA. Corresponding opposite effects were achieved by the lentivirus-mediated knock-down of miR-143, such as upregulation of CAR, CYP2B6 and CYP2C8 mRNAs as measured after five days of infection (Fig. 5). 
Thomas et al.: A Lentivirus-Based System to Functionally Silence Nuclear Receptors in Primary Human Hepatocytes

Table 1. Short summary of the protocol for the generation and concenrtration of lentiviral particles. "S" means that these steps have to be performed under higher biological safety conditions

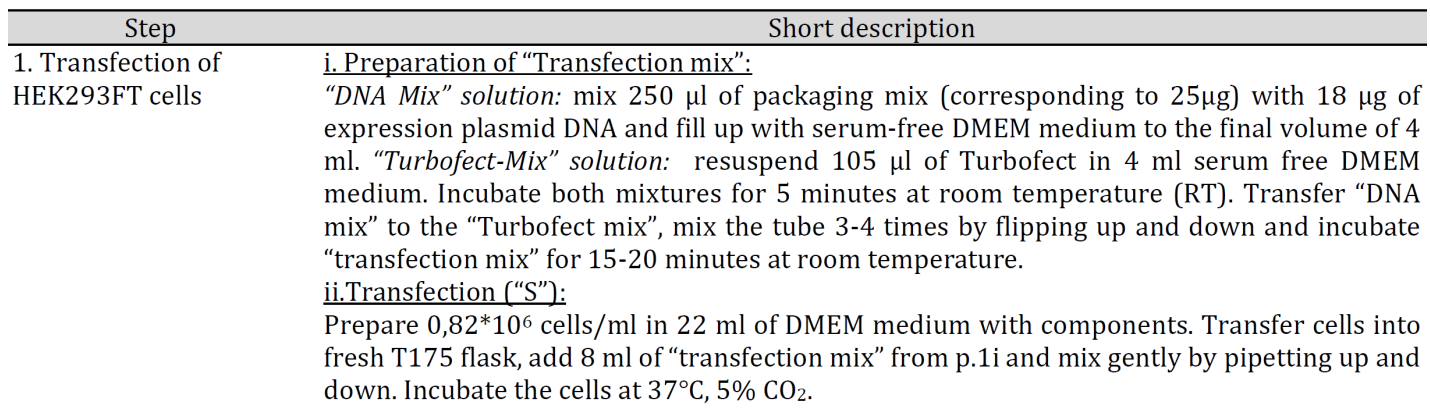

2. First harvesting of supernatant ( $48 \mathrm{~h}$ after transfection) ("S").
A. First option: using ultracentrifuge.

i. Collecting the supernatants

Transfer the culture medium (ca. $30 \mathrm{ml}$ ) into $50 \mathrm{ml}$ Falcon tube. Add $30 \mathrm{ml}$ of fresh medium to the cells for further incubation at $37^{\circ} \mathrm{C}$. Centrifuge the transferred medium at $1750 \mathrm{xg}$ for $5 \mathrm{~min}$ at $4^{\circ} \mathrm{C}$ to pellet cells or cellular debris. Filter the supernatant through $0,45 \mu \mathrm{m}$ PVDF into ultracentrifuge tubes. ii. Centrifugation using ultracentrifuge

Centrifuge supernatant at $50.000 \times \mathrm{g}$ for $90 \mathrm{~min}$ at $4^{\circ} \mathrm{C}$.

iii. Dissolving the viral pellet

After centrifugation, discard the supernatant, let the pellet dry at RT and resuspend it in 50 $\mu \mathrm{l} 1 \%$ BSA/PBS. To completely dissolve the pellet, keep the tubes in a vertical position at $4^{\circ} \mathrm{C}$ at least overnight. Store at $-80^{\circ} \mathrm{C}$.

B. Alternative option: polyethylene glycol precipitation using PEG-it ${ }^{T M}$

i. Collecting the supernatants

As described above in $2 \mathrm{Ai}$. ii. Precipitation with PEG-it ${ }^{\mathrm{TM}}$

Add $6 \mathrm{ml}$ of $5 \mathrm{xPEG}$-it solution to the filtered supernatant from $2 \mathrm{Ai}$, mix by flicking up and down and store at $4^{\circ} \mathrm{C}$ for at least overnight to precipitate viral particles.

3. Second harvesting of supernatant $(72 \mathrm{~h}$ after transfection) ("S").
A. First option: using ultracentrifuge

Repeat the steps $2 \mathrm{Ai}$-iii as described above

B. Alternative option: using PEG-it ${ }^{T M}$

Repeat the steps $2 \mathrm{Bi}-\mathrm{ii}$ as described above

iii. Pelleting viral particles after PEG-it ${ }^{\mathrm{TM}}$ precipitation.

Centrifuge the precipitated supernatants from steps $2 \mathrm{~B} i i$

and $3 \mathrm{Bii}$ at $1750 \mathrm{xg}$ for $30 \mathrm{~min}$ at $4^{\circ} \mathrm{C}$. Resuspend it in $50 \mu \mathrm{l} 1 \% \mathrm{BSA} / \mathrm{PBS}$. Store at $-80^{\circ} \mathrm{C}$.

\section{Detailed protocol}

The short protocol for the generation and concentration of lentiviral particles is summarized in Table 1. A common challenge of the virus production part concerns the concentration of viral particles which is critical for high infection rates. Because some labs may not have the opportunity to install an ultracentrifuge under "safety standard level" conditions, an alternative protocol is provided for virus precipitation using polyethylene glycol. Whereas the final outcome does not influence the quality of viral particles, the ultracentrifuge protocol is certainly less time- and cost- consuming. Both workflows were successfully applied and validated in our lab and a comparison of the two protocols is presented in Table 2.

The whole procedure of virus production with subsequent titer determination takes approximately six to ten working days. The measureable effects of gene targeting following viral infection of hepatocytes takes approximately three to five days. The properly frozen and aliquoted viral stocks have been tested and showed no decrease in titer and infection capacity over a long period of time. The detailed protocol with step-by-step description of the procedure can be found here: http://seek.virtuelle-leber.de/sops/13. 
Thomas et al.: A Lentivirus-Based System to Functionally Silence Nuclear Receptors in Primary Human Hepatocytes

Table 2. Overview of two different methods for the purification of viral particles. Comparison of protocols using ultracentrifuge (option A) and polyethylene glycol (PEG-it ${ }^{\mathrm{TM}}$ ) precipitation (option B)

\begin{tabular}{|c|c|c|}
\hline & "Ultracentrifuge" protocol & "PEG-it" protocol \\
\hline Time consumption & 6 working days & 7 working days \\
\hline Average titer $(\mathrm{TU} / \mathrm{ml})$ & $\sim 4 \times 10^{7}$ & $\sim 0,6 \times 10^{7}$ \\
\hline $\begin{array}{l}\text { MOI to be used for successful } \\
\text { infection of phh }\end{array}$ & MOI 3 & MOI 3 \\
\hline Operating procedure & -Requires skill in handling ultracentrifuge & simple \\
\hline Costs & $\begin{array}{l}\text { - } \quad \text { One-time costs for ultracentrifuge } \\
\text { Repeated costs for acquisition of one- } \\
\text { time use ultracentrifuge tubes }\end{array}$ & - Repeated costs for acquisition of PEG-it ${ }^{\mathrm{TM}}$ \\
\hline General note & $\begin{array}{l}\text { Preferred way to establish routine production } \\
\text { of pure virus with high titer with minimal } \\
\text { operating expense }\end{array}$ & $\begin{array}{l}\text { Suitable for application in low-scale } \\
\text { experiments or when no ultracentrifuge is } \\
\text { available in S2 lab }\end{array}$ \\
\hline
\end{tabular}

\section{Conclusions}

In summary, we developed robust, comprehensive and reliable protocols for the design, generation and purification of lentiviral particles and their application for transduction of $\mathrm{PHH}$. Alternative protocols for implementation depending on the available lab equipment are provided. This method has important advantages compared with alternative approaches. As lentiviral infection does not appear to affect cellular viability and hepatic phenotype, it is the method of choice to carry out prolonged or stable downregulation not only of nuclear receptors but also other target genes in PHH and can be easily adapted to other primary human cells and cell culture models.

For the protocol validation, we generated a panel of vectors coding for active shRNAs targeting major nuclear receptors as well as known liver-specific microRNAs and antimicroRNAs. We demonstrated that delivery of RNAi molecules via lentivirus represents an efficient and simple method for the transfection of PHH to study molecular mechanisms of NR functions. Furthermore, we could demonstrate that lentivirus-mediated overexpression of miR-143 as well as downregulation of miR-143 expression using anti-miR-143 affects expression of CAR as well as its target genes, CYP2B6 and CYP2C8. This interesting observation warrants further investigation of the molecular mechanisms underlying miR143 mediated regulation of drug-metabolizing enzymes in PHH.

\section{Abbreviations}

CITCO (6-(4-Chlorophenyl)imidazo(2,1-b)(1,3)thiazole-5-carbaldehyde 0-(3,4dichlorobenzyl)oxime); CAR (Constitutive androstane receptor); LDH (Lactate dehydrogenase); MOI (Multiplicity of infection); NR (Nuclear receptor); PPAR $\alpha$ (Peroxisome proliferator receptor alpha); PHH - (Primary human hepatocytes); shRNAs (small hairpin RNAs).

\section{Disclosure Statement}

The authors declare that they have no competing financial interests. 
Thomas et al.: A Lentivirus-Based System to Functionally Silence Nuclear Receptors in Primary Human Hepatocytes

\section{Acknowledgements}

The EGFP-expressing lentiviral vector pLenti6-EGFP was kindly provided by Dr. Martin Kriebel, NMI, Reutlingen. We thank Igor Liebermann and Britta Klumpp, Stuttgart, for expert technical assistance. For isolation and delivery of human primary hepatocytes, we would like to thank the lab members of Wolfgang Thasler (Munich), Andreas Nüssler (Tuebingen) and Thomas Weiss (Regensburg). This study was supported by the German BMBF (Virtual Liver grant 0315755) and by the Robert Bosch Foundation, Stuttgart, Germany.

\section{References}

1 Wilson RC, Doudna JA: Molecular Mechanisms of RNA Interference. Annual Review of Biophysics 2013;42:217-239.

2 Pecot CV, Calin GA, Coleman RL, Lopez-Berestein G, Sood AK: RNA interference in the clinic: challenges and future directions. Nat Rev Cancer 2011;11:59-67.

-3 Ilan Y, Saito H, Thummala NR, Chowdhury NR: Adenovirus-mediated gene therapy of liver diseases. Semin. Liver Dis 1999;19:49-59.

4 Li H, Fu X, Chen Y, Hong Y, Tan Y, Cao H, Wu M, Wang H: Use of Adenovirus-Delivered siRNA to Target Oncoprotein p28GANK in Hepatocellular Carcinoma. Gastroenterology 2005;128:2029-2041.

-5 Rana R, Surapureddi S, Kam W, Ferguson S, Goldstein JA: Med25 Is Required for RNA Polymerase II Recruitment to Specific Promoters, Thus Regulating Xenobiotic and Lipid Metabolism in Human Liver. Mo. Cell Biol 2011;31:466-481.

6 Jover R, Bort R, Gómez-Lechón MJ, Castell JV: Cytochrome P450 regulation by hepatocyte nuclear factor 4 in human hepatocytes: a study using adenovirus-mediated antisense targeting. Hepatology 2001;33:668-675.

7 Grimm D, Kay MA: Combinatorial RNAi: a winning strategy for the race against evolving targets? Mol Ther 2007;15:878-888.

8 Leonard JN, Schaffer DV: Antiviral RNAi therapy: emerging approaches for hitting a moving target. Gene Ther 2006;13:532-540.

9 Gregory SM, Nazir SA, Metcalf JP: Implications of the innate immune response to adenovirus and adenoviral vectors. Future Virol 2011;6:357-374.

$\checkmark 10$ Ferry N, Duplessis O, Houssin D, Danos 0, Heard JM: Retroviral-mediated gene transfer into hepatocytes in vivo. Proc Natl Acad Sci USA 1991;88:8377-8381.

11 Bos T, De Bruyne E, Heirman C, Vanderkerken K: In Search of the Most Suitable Lentiviral shRNA System. Curr Gene Ther 2009;9:192-211.

12 Follenzi A, Santambrogio L, Annoni A: Immune Responses to Lentiviral Vectors. Current Gene Ther 2007;7:306-315.

13 Rana R, Chen Y, Ferguson SS, Kissling GE, Surapureddi S, Goldstein JA: Hepatocyte Nuclear Factor 4? Regulates Rifampicin-Mediated Induction of CYP2C Genes in Primary Cultures of Human Hepatocytes. Drug Metab Dispos 2010;38:591-599.

14 Zamule SM, Strom SC, Omiecinski CJ: Preservation of Hepatic Phenotype in Lentiviral-Transduced Primary Human Hepatocytes. Chem Biol Interact 2008;173:179-186.

15 Klein K, Thomas M, Winter S, Nussler AK, Niemi M, Schwab M, Zanger UM: PPARA: A Novel Genetic Determinant of CYP3A4 In Vitro and In Vivo. Clinical Pharmacol Therp 2012;91:1044-1052.

16 Thomas M, Burk O, Klumpp B, Kandel BA, Damm G, Weiss TS, Klein K, Schwab M, Zanger UM: Direct transcriptional regulation of human hepatic cytochrome P450 3A4 (CYP3A4) by peroxisome proliferatoractivated receptor alpha (PPAR $\alpha$ ). Mol Pharmacol 2013;83:709-718. 
Thomas et al.: A Lentivirus-Based System to Functionally Silence Nuclear Receptors in Primary Human Hepatocytes

17 Godoy P, Hewitt NJ, Albrecht U, Andersen ME, Ansari N, Bhattacharya S, Bode JG, Bolleyn J, Borner C, Böttger J, Braeuning A, Budinsky RA, Burkhardt B, Cameron NR, Camussi G, Cho C-S, Choi Y-J, Craig Rowlands J, Dahmen U, Damm G, Dirsch O, Donato MT, Dong J, Dooley S, Drasdo D, Eakins R, Ferreira KS, Fonsato V, Fraczek J, Gebhardt R, Gibson A, Glanemann M, Goldring CEP, Gómez-Lechón MJ, Groothuis GMM, Gustavsson L, Guyot C, Hallifax D, Hammad S, Hayward A, Häussinger D, Hellerbrand C, Hewitt P, Hoehme S, Holzhütter H-G, Houston JB, Hrach J, Ito K, Jaeschke H, Keitel V, Kelm JM, Kevin Park B, Kordes C, Kullak-Ublick GA, LeCluyse EL, Lu P, Luebke-Wheeler J, Lutz A, Maltman DJ, Matz-Soja M, McMullen P, Merfort I, Messner S, Meyer C, Mwinyi J, Naisbitt DJ, Nussler AK, Olinga P, Pampaloni F, Pi J, Pluta L, Przyborski SA, Ramachandran A, Rogiers V, Rowe C, Schelcher C, Schmich K, Schwarz M, Singh B, Stelzer EHK, Stieger B, Stöber R, Sugiyama Y, Tetta C, Thasler WE, Vanhaecke T, Vinken M, Weiss TS, Widera A, Woods CG, Xu JJ, Yarborough KM, Hengstler JG: Recent advances in 2D and 3D in vitro systems using primary hepatocytes, alternative hepatocyte sources and non-parenchymal liver cells and their use in investigating mechanisms of hepatotoxicity, cell signaling and ADME. Arch Toxicol 2013;87:1315-1530.

18 Hofmann MH, Blievernicht JK, Klein K, Saussele T, Schaeffeler E, Schwab M, Zanger UM: Aberrant splicing caused by single nucleotide polymorphism c.516G>T [Q172H], a marker of CYP2B6*6, is responsible for decreased expression and activity of CYP2B6 in liver. J Pharmacol Exp Ther 2008;325:284-292.

19 Feidt DM, Klein K, Nüssler A, Zanger UM: RNA-interference approach to study functions of NADPH : cytochrome P450 oxidoreductase in human hepatocytes. Chem Biodivers 2009;6:2084-2091.

-20 Feidt DM, Klein K, Hofmann U, Riedmaier S, Knobeloch D, Thasler WE, Weiss TS, Schwab M, Zanger UM: Profiling induction of cytochrome p450 enzyme activity by statins using a new liquid chromatographytandem mass spectrometry cocktail assay in human hepatocytes. Drug Metab. Dispos 2010;38:1589-1597.

21 Rieger JK, Bodan DA, Zanger UM: MIRNA-DISTILLER: A Stand-Alone Application to Compile microRNA Data from Databases. Front Genet 2011;2:39.

-22 Rieger JK, Klein K, Winter S, Zanger UM: Expression variability of absorption, distribution, metabolism, excretion-related microRNAs in human liver: influence of nongenetic factors and association with gene expression. Drug Metab. Dispos 2013;41:1752-1762.

-23 Zhao X, Yang Z, Li G, Li D, Zhao Y, Wu Y, Robson SC, He L, Xu Y, Miao R, Zhao H: The role and clinical implications of microRNAs in hepatocellular carcinoma. Sci China Life Sci 2012;55:906-919. 